\title{
REMARKS ON THE HEAWOOD CONJECTURE (NONORIENTABLE CASE)
}

\author{
BY J. W. T. YOUNGS
}

\author{
Communicated by J. D. Swift, August 22, 1967
}

1. Introduction. In 1890 Heawood [3] stated a problem which has become known as the Heawood map-coloring conjecture. It is concerned with the chromatic number of 2-manifolds, and is one of the oldest problems in combinatorics.

The conjecture for orientable manifolds is not yet completely solved, though considerable progress has been made in the past few years. On the other hand, the companion conjecture for nonorientable manifolds was solved completely by Ringel [4] during the years 1953-1959. For this purpose he developed a theory of "leading" permutations but is hardly pleased with the complications involved. The object of this note is to announce a successful attack on the problem using quite simple combinatorial techniques.

2. Definitions, theorems and conjectures. All manifolds $\tilde{M}$ are closed, nonorientable and of dimension 2. Each $\tilde{M}$ has a standard model which is a 2-sphere with added cross caps. $\gamma(\tilde{M})$, the genus of $\tilde{M}$, is the number of cross caps. $K_{n}$ is the complete $n$-graph, that is, the graph with $n$ vertices where each pair of distinct vertices is joined by exactly one arc. $\tilde{\gamma}(K)$, the nonorientable genus of $K$, is the smallest integer $k$ such that the graph $K$ can be topologically imbedded in a manifold of genus $k$. The chromatic number of $\tilde{M}$ is designated by $\operatorname{ch}(\tilde{M})$. Define

$$
\begin{gathered}
\tilde{H}(q)=\left[\left(7+(1+24 q)^{1 / 2}\right) / 2\right],{ }^{1} \quad q=1,2,3, \cdots, \\
\tilde{I}(n)=\{(n-3)(n-4) / 6\}, \quad n=5,6,7, \cdots .
\end{gathered}
$$

The Heawood theorem states that

$$
\operatorname{ch}(\tilde{M}) \leqq \tilde{H}(\gamma(\tilde{M}))
$$

and the Heawood conjecture is that equality holds in (1).

The complete graph theorem is that

$$
\tilde{\gamma}\left(K_{n}\right) \geqq I(n),
$$

and the complete graph conjecture is that equality holds in (2).

$1[a]$ is the largest integer not greater than $a$, and $\{a\}$ is the smallest integer not less than $a$. 
TheOREM (RINGeL). The Heawood conjecture is true unless $\tilde{M}$ is a Klein bottle, in which case $6=\operatorname{ch}(\tilde{M})<\tilde{H}(\gamma(\tilde{M}))=\widetilde{H}(2)=7 .^{2}$

An equivalent result is the following

ThEOREM (RINGEL). The complete graph conjecture is true for $n \geqq 5$ unless $n=7$, in which case $\tilde{\gamma}\left(K_{7}\right)=3>2=I(7)$.

3. General comments. In addition to $K_{n}$ we shall deal with a graph which is nearly complete. Take $K_{n}$ and add two distinct vertices $x$ and $y$ to it. Now join $x$ and $y$ to all the vertices of $K_{n}$. The resulting graph, designated by $K_{n+2}^{2}$, fails to be complete merely because $x$ and $y$ are not joined by an arc.

If a graph $K$ is imbedded in $\tilde{M}$ and $\tilde{\gamma}(K)=\gamma(\tilde{M})$, then it can be shown that each component of $(M-K)$ is a 2-cell and hence the Euler formula may be applied. (See [6].) If $N_{k}$ is the number of $k$-sided cells in the imbedding, and $K=K_{n}$, then $\tilde{\gamma}\left(K_{n}\right)=(n-3)(n-4) / 6$ $+\frac{1}{3} \sum_{k>3}(k-3) N_{k}$. Hence $\tilde{\gamma}\left(K_{n}\right) \geqq\{(n-3)(n-4) / 6\}=\tilde{I}(n)$. This suggests that to show

$$
\tilde{\gamma}\left(K_{n}\right)=\tilde{I}(n)
$$

may involve six cases depending upon the membership of $n$ in the various residue classes modulo 6 . This expectation is realized for $n \equiv 3,4$ and $5(\bmod 6)$ and the examples presented are restricted to these cases.

If $n \equiv 3$ or $4(\bmod 6)$, then $(n-3)(n-4) \equiv 0(\bmod 6)$. In each of these cases we exhibit a triangular imbedding of $K_{n}$ (that is, one for which $N_{k}=0$ if $k>3$ ) in a manifold $\tilde{M}$, and thus prove (3).

If $n \equiv 5(\bmod 6)$, then $(n-3)(n-4) \equiv 2(\bmod 6)$ and a triangular imbedding of $K_{n}$ is impossible. To get around this difficulty we exhibit a triangular imbedding of $K_{n}^{2}$ in a manifold $\tilde{N}$ of genus $\tilde{I}(n)-1$. By adding one suitably positioned cross cap to $\tilde{N}$ we get a manifold $\tilde{M}$ containing $K_{n}^{2}$ such that the exceptional vertices $x$ and $y$ can be joined on $\tilde{M}$ by an arc which does not intersect $K_{n}^{2}$. Hence (3) is proved.

Some explanation is needed for the terms "we exhibit a triangular imbedding" used above. If $K$ is a connected graph with no multiple edges or loops, and vertices $v_{1}, \cdots, v_{n}$, then a schema is an array $S:\left[v_{i}, P_{v_{i}}\right], i=1, \cdots, n$, where $P_{v_{i}}$ is a cyclic permutation of the vertices which are joined to $v_{i}$.

The schema satisfies $R$ at $(g, h)$ if $P_{g}^{-1}(h)=P_{h}^{-1}(g)$ and $P_{g}(h)$ $=P_{h}(g)$; it satisfies $R^{*}$ at $(g, h)$ if $P_{g}^{-1}(h)=P_{h}(g)$ and $P_{g}(h)=P_{h}^{-1}(g)$.

If $S$ satisfies $R$, or $R^{*}$ at $(g, h)$, then it satisfies it at $(h, g)$ so that

\footnotetext{
${ }^{2}$ See Franklin [1].
} 
the conditions are really on unordered pairs of distinct vertices of $K$. Designate the totality of unordered pairs of vertices, each of which determines an arc of $K$, by $A$. Given $S$, let $R_{S}\left(R_{S}^{*}\right)$ be the subset of $A$ where $R\left(Q^{*}\right)$ is satisfied.

Ringel has shown that if $R_{S}^{*}=A$, then $S$ gives a precise receipt for a triangular imbedding of $K$ in an orientable manifold. Moreover, $S$ determines a triangular imbedding of $K$ in a nonorientable manifold if (i) $R_{S} \cup R_{S}^{*}=A$.

(ii) No modification of $S$, made by replacing certain permutations $P_{x}$ by $P_{x}^{-1}$ produces a schema $T$ for which $R_{T}^{*}=A$.

In the first case he calls $S$ an orientable, in the second, a nonorientable schema for $K$.

4. Construction of nonorientable schemas. The basic concepts employed, such as singular arc, rotation, circuit, current graph, etc., have been employed in [5] and are not redefined here. ([2] and [7] are also suggested as general references.)

EXAMPLE 1. $K_{n}^{2}, n=6 t+5$, using the symbols $x$ and $y$ in addition to the elements of $Z_{6 t+3}$ to name the vertices.

A solution is given for $t=2$ in Figure 1 . The currents are from $Z_{15} \backslash 0$, and, in order to make the representation possible on a plane, the arcs carrying currents 3 and 6 have been cut.

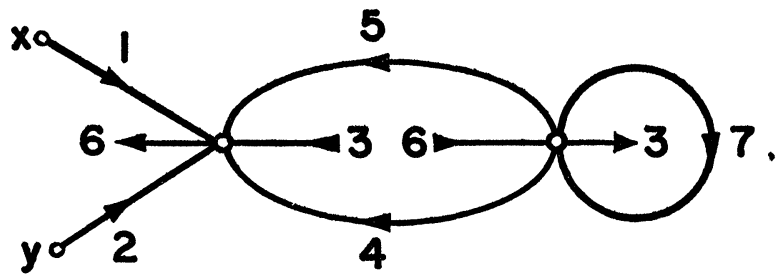

FIGURE 1

All the rotations are counterclockwise, and we get a single circuit. The currents on the directed arcs of the circuit provide a "provisional" cyclic permutation $P_{0}^{\prime}$ of $Z_{15} \backslash 0$; namely, $(7,3,-5,-6,-2,2$, $-4,-7,5,-1,1,6,4,-3)$. The diagram suggests that to get the permutation $P_{0}$ of all the vertices of $K_{n}^{2}$ joined to 0 we insert $x$ between -1 and 1 and $y$ between -2 and 2 in $P_{0}^{\prime}$ to obtain

$$
0.7,3,-5,-6,-2, y, 2,-4,-7,5,-1, x, 1,6,4,-3 \text {. }
$$

For each element $g$ in $Z_{15}$ the permutation $P_{g}$ is obtained by an $a d d i$ tivity principle. Without changing the order, add $g$ to each group 
element in (4). Define $P_{x}$ to be $(0,-1,-2, \cdots)$ and $P_{y}$ to be $(0,-2,-4, \cdots)$.

The result is a nonorientable schema for $K_{17}^{2}$. What guarantees this is that: (i) At the two vertices of degree 6 one has $1+5=2+4=6$, $5+7+3=0$ and $4+3=7$. (ii) The elements -1 and -2 generate $Z_{15}$.

There is a very useful nomogram (Figure 2) from which all this information may be read at a glance.

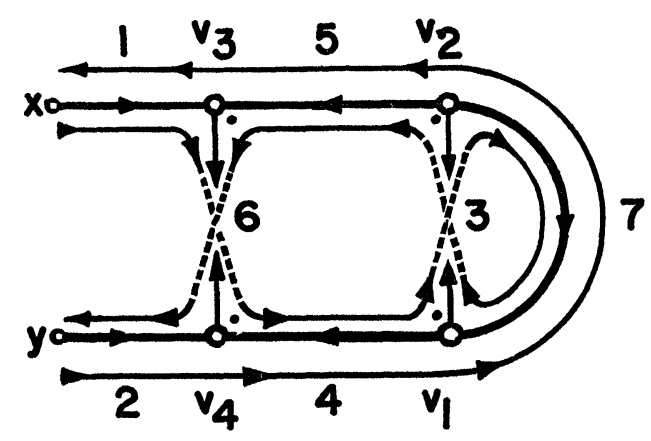

FIGURE 2

This is not a current graph since, for example, the two directed "pseudo arcs" carrying current 6 do not have "terminal" vertices. Observe, however, that Kirchhoff's current law holds at $v_{1}, v_{2}, v_{3}$ and $v_{4}$ and is precisely the collection of requirements (i) above. This nomogram, therefore, certainly simplifies checking the four requirements in (i). In addition, however, it can be used to obtain the single circuit of Figure 1 so that Figure 2 carries all the information for a nonorientable imbedding of $K_{17}^{2}$. The circuit is reconstructed as follows.

Start with the directed arc carrying the current 7 and follow the path indicated in the figure. This leads us to $v_{1}$ and up the pseudo arc with current 3 . Record 3 as following 7 . This agrees with (4). From $v_{2}$ the path goes to $v_{3}$ but in doing so it takes a clockwise rotation at $v_{2}$ where a counterclockwise rotation is called for. Notice that there is a dot in the angle where the rotation is negated. As a mnemonic device, negate the current 5 which one might expect to be recorded next, and instead record -5 . So far the permutation is $7,3,-5$, which agrees with (4). Following the negating rule wherever necessary along the indicated path we reproduce (4) thus making diagrams of the type shown in Figure 1 unnecessary in the future.

In addition, we get some precise information about requirements $R$ and $R^{*}$. The vertices $v_{1}, v_{2}, v_{3}, v_{4}$ are said to determine a "dotted" box. 
THEOREM. If $g$ is found on a dotted box, then $R$ holds at $(0, g)$; if $g$ is not found on a dotted box, then $R^{*}$ holds at $(0, g)$.

Diagrams of the type found in Figures 1 and 2 are called cascades. In this fashion we find triangular nonorientable imbeddings of $K_{6 t+5}^{2}$ and prove $(3)$ for $n \equiv 5(\bmod 6)$.

The continuation is quite simple as shown in Figure 3 for $t=5$.

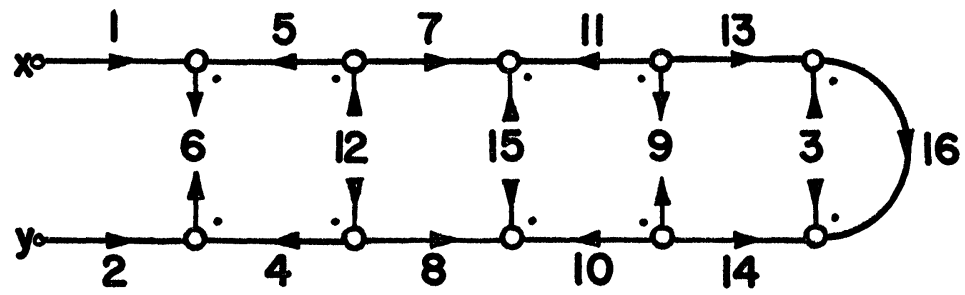

FiguRE 3

EXAmple 2. $K_{n}, n=6 t+4$, using the symbol $z$ in addition to the elements of $Z_{6 t+8}$ to name the vertices.

A solution is given for $t=2$ in Figure 4. Note that the first two leafs are duplications of the cascade in Figure 2 while the third is obtained by reversing all the currents and turning the diagram upside down. There are three circuits labelled [0], [1], [2], and the group element recorded where circuit $[a]$ "meets" circuit $[b]$ is from the coset $[b-a]$. (See [7].) Moreover, Kirchhoff's current law holds at all vertices except $B$ wherein there is a "vortex" of -3 . Finally each vertex is of degree 3 . Each circuit $[a]$ provides a cyclic permutation $\{a\}$ of $Z_{15} \backslash 0$ and $z$, where $z$ is inserted in $\{0\}$, for example, between 1 and 2 as suggested by the position of the letter $z$ in the vortex -3 at $B$. If $g \equiv a(\bmod 3)$, then $P_{g}$ is obtained from $\{a\}$ by the additivity principle, and $P_{z}$ is $(2,0,1 ; 14,12,13 ; 11,9,10 ; 8,6,7 ; 5,3,4)$. A general theorem gurantees that we obtain a nonorientable schema for $K_{16}$.

EXAMPLE 3. $K_{n}, n=6 t+3$, using the elements of $Z_{6 t+3}$ to name the vertices.

A solution is given for $t=2$. There are three leafs as in Figure 4 but there is a change in leaf 3 which is now obtained from leaf 2 by multiplying the currents there by -2 . There is now no "vortex" at $B$ and hence no exceptional vertex $z$. A general theorem guarantees that on proceeding as in Example 2 we obtain a nonorientable schema for $K_{15}$.

The last two examples, with three "generating" permutations are said to be of index 3 ; the first is of index 1 .

The equality (3) is proved in this fashion for $n \equiv 3,4$ and $5(\bmod 6)$ 
Currents from $Z_{15}$
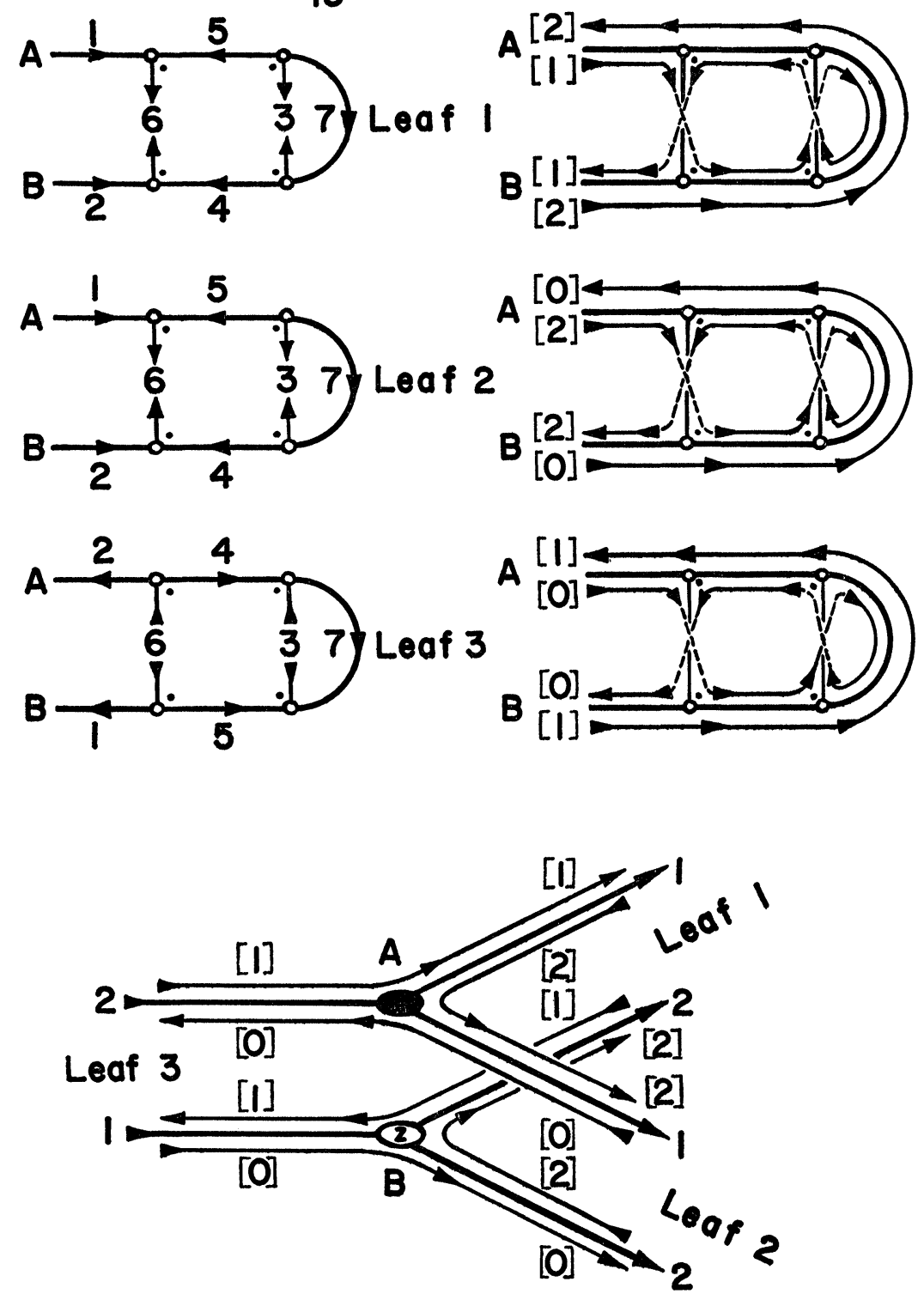

FIGURE 4 
by employing some fortunate combinatorial properties of the cyclic group $Z_{6 t+3}$. No such extreme unification appears possible if $n \equiv 0,1$ and $2(\bmod 6)$.

\section{REFERENCES}

1. Philip Franklin, A six color problem, J. Math. and Phys. 13 (1934), 363-369.

2. P. J. Heawood, Map-color theorem, Quart. J. Math. Oxford Ser. 24 (1890), 332338.

3. Gerhard Ringel, Färbungsprobleme auf Flächen und Graphen, Deutscher Verlag der Wiss. Berlin, 1959.

4. C. M. Terry, L. R. Welch and J. W. T. Youngs, The genus of $K_{128,}$ J. Comb. Th., 2 (1967), 43-60.

5. J. W. T. Youngs, Minimal imbeddings and the genus of a graph, J. Math. Mech. 12 (1963), 303-315.

6. - "The Heawood map-coloring conjecture," in Graph theory and theoretical physics, Academic Press, London, 1967, pp. 313-354.

7. William Gustin, Orientable embedding of Cayley graphs, Bull. Amer. Math. Soc. 69 (1963), 272-275.

Untversity of California, Santa Cruz 